\title{
Estudo comparativo da estrutura fitossociológica de dois fragmentos de Caatinga em níveis diferentes de conservação
}

\author{
João Tavares Calixto Júnior'1, Marcos Antônio Drumond² \\ ${ }^{1}$ Faculdade de Juazeiro do Norte, Rua São Francisco, n 1.224, São Miguel, CEP 58.900-970, Juazeiro do Norte, CE, Brasil \\ ${ }^{2} E$ mbrapa Semi-Árido, BR 428, Km 152, CP 23, Zona Rural, CEP 56.302-970, Petrolina, PE, Brasil
}

"Autor correspondente:

joaojrbio@gmail.com

Termos para indexação:

Vegetação caducifólia espinhosa

Fitossociologia

Sucessão ecológica

Index terms:

Thorny deciduous vegetation

Phytosociology

Ecological sucession

Histórico do artigo:

Recebido em 12/03/2014

Aprovado em 11//12/2014

Publicado em 31/12/2014

doi: 10.4336/2014.pfb.34.80.670
Resumo - Objetivou-se neste trabalho, o comparativo da estrutura fitossociológica de dois fragmentos de Caatinga com níveis de sucessão ecológica primária e secundária localizados na Embrapa Semiárido, Petrolina, PE. No levantamento florístico utilizouse o método das parcelas, onde foram plotadas 10 unidades amostrais de $8 \mathrm{~m} \mathrm{x} 40 \mathrm{~m}$ distribuídas de forma aleatória. Todos os indivíduos vivos com diâmetro ao nível do solo (DNS) $\geq 3 \mathrm{~cm}$ foram inventariados, sendo medidas também as suas alturas totais. Observaram-se diferenças significativas na estrutura diamétrica, mas a distribuição da frequência hipsométrica mostrou que a maioria dos indivíduos nas duas áreas apresentou pequeno porte $(<5 \mathrm{~m})$. Através da análise de similaridade e das coordenadas principais observou-se a formação de três grupos distintos quando comparadas a outros levantamentos em Caatinga sensu stricto. Os resultados evidenciaram que as áreas estudadas apresentam riqueza de espécies compatível a ambientes de Caatinga, que os fragmentos se encontram em estádio inicial (área I) e intermediário de sucessão (área II) e que 30 anos de regeneração não foram suficientes para a área I alcançar um nível de desenvolvimento ecológico semelhante à área melhor conservada.

\section{Comparative study of phytosociological structure of two fragments of Caatinga in different levels of conservation}

\begin{abstract}
The objective of this work was the comparison of vegetation structure of two Caatinga fragments with primary and secondary levels of ecological succession, located at Embrapa Semiarido, Petrolina, State of Pernambuco, Brazil. For floristic survey it was used the method of plots, where 10 sampling units of $8 \mathrm{~m}$ x $40 \mathrm{~m}$ randomly distributed were plotted. All living individuals with diameter at ground level (DGL) $\geq 3 \mathrm{~cm}$ were inventoried and also measured their total heights. We observed significant differences in the diametric structure, but the distribution of hypsometric frequency showed that the majority of individuals in both areas were small-sized $(<5 \mathrm{~m})$. Through similarity analysis and principal coordinates, we observed the formation of three distinct groups when compared to other surveys in Caatinga sensu stricto. The results showed that the studied areas have floristic richness compatible with Caatinga environments, the fragments are in early stages (area I) and intermediate succession (area II) and 30 years of regeneration were not enough for area I to achieve a level of development similar to the ecologically better conserved area.
\end{abstract}




\section{Introdução}

O bioma Caatinga, segundo Andrade et al. (2005) é o maior e mais importante da Região Nordeste do Brasil, estendendo-se pelo domínio de climas semiáridos, numa área de quase 100 milhões de hectares, o que equivale a cerca de $11 \%$ do território nacional. Está compreendido entre os paralelos de $2^{\circ} 54^{\prime} \mathrm{S}$ e $17^{\circ} 21^{\prime}$ W e, de acordo com Prado (2003), abrange parte dos estados do Piaú, Ceará, Rio Grande do Norte, Paraíba, Pernambuco, Alagoas, Sergipe, Bahia e Minas Gerais. Para Pessoa et al. (2008) e Pereira Júnior et al. (2012), a Caatinga é um bioma rico em recursos genéticos, dado a sua alta biodiversidade quando comparada a outras regiões semiáridas no mundo. Apresenta uma variada cobertura vegetal, em grande parte determinada pelo clima, relevo e embasamento geológico, que em suas múltiplas inter-relações, resultam em sistemas ecológicos bastante variados (Andrade-Lima, 1981). Em escala local, variações topográficas no seu interior contribuem para a ocorrência de gradientes menores. Além disso, diferenças litológicas, tanto em escala regional como local, contribuem para o diversificado conjunto vegetacional da província das caatingas (Rodal et al., 2008). Em virtude das condições climáticas, a vegetação endêmica é ramificada, com aspecto arbustivo, apresentando folhas pequenas ou modificadas em espinhos, de modo a evitar a evapotranspiração, ocorrendo a perda de folhas na época seca (Souto, 2006).

De acordo com Araújo (2007) o bioma Caatinga é, provavelmente, o menos estudado em relação à flora e à fauna e um dos que têm sofrido maior degradação, pelo uso desordenado e predatório, nos últimos 400 anos.

A Caatinga tem sido historicamente devastada para ceder lugar a atividades agropecuárias que ocupam várias extensões no semiárido. Uma vez abandonada a exploração dessas áreas, tem início o processo de sucessão ecológica, quase sempre interrompido por novas intervenções. Assim, a vegetação da Caatinga apresenta-se como um mosaico formado por variados estágios seriais, resultante dos usos ali imputados (Andrade et al., 2007). Calixto Júnior \& Drumond (2011) informam não serem muitos os estudos que investigam aspectos de sucessão ecológica em ambientes de Caatinga.

Diante desta realidade, vários autores atentam para a necessidade urgente do conhecimento e da conservação do referido bioma (Araújo \& Ferraz, 2008; Rodal et al., 2008; Souza \& Rodal, 2010; Pereira Júnior et al., 2012). Neste contexto, este trabalho teve como objetivo a comparação de duas fitofisionomias de Caatinga com diferentes estados de conservação e, também, sua comparação com outros levantamentos realizados em áreas de Caatinga sensu stricto, com semelhantes históricos de uso e/ou estados de conservação. Com isto, procura-se compreender melhor o comportamento da Caatinga diante de ações de degradação a ela imputadas, visando à melhor manutenção e um maior conhecimento sobre a sua diversidade biológica, principalmente da flora lenhosa em áreas de Caatinga com níveis diferentes de intervenção antrópica, e sob estádios distintos de sucessão ecológica.

\section{Material e métodos}

\section{Área de estudo}

$\mathrm{O}$ estudo foi realizado em duas áreas de vegetação caducifólia espinhosa (Caatinga) pertencentes à Embrapa Semiárido (09 $09^{\circ} \mathrm{S}, 42^{\circ} 22^{\prime} \mathrm{W}$ ) localizada a $42 \mathrm{~km}$ da sede de Petrolina, numa altitude média de 380 m (Calixto Júnior \& Drumond, 2011). O Município de Petrolina está localizado na Mesorregião do São Francisco, extremo sudoeste do Estado de Pernambuco, Nordeste do Brasil. De acordo com a classificação climática de Köppen, o clima das áreas é do tipo BSwh', definido como semiárido quente, de vegetação xerófila. A temperatura média varia de $20{ }^{\circ} \mathrm{C}$ a $38{ }^{\circ} \mathrm{C}$, sendo outubro o mês mais quente e julho o mais frio. As áreas apresentam alta insolação, com média anual de $2.800 \mathrm{~h}$, e média de evapotranspiração de $2.000 \mathrm{~mm} \cdot \mathrm{ano}^{-1}$.

A área I $\left(09^{\circ} 04^{\prime} 53^{\prime \prime} \mathrm{S}, 40^{\circ} 32^{\prime} 43^{\prime \prime} \mathrm{W}\right)$ possui um contorno regular e está distribuída em uma área com cerca de $2 \mathrm{ha}$, com solo caracterizado por apresentar muitos fragmentos de rocha. Foi submetida à corte raso em toda a sua extensão no ano de 1979, desde então, recuperando-se sem intervenção antrópica. A área foi cercada para impedir a entrada de animais não silvestres.

A área II dista cerca de $1 \mathrm{~km}$ da área I e após analise do histórico de uso, foi observado que se trata de uma área em bom estado conservação, não havendo notícias de intervenção antrópica acentuada após a instalação da Estação Experimental da Embrapa Semiárido, por volta de 1978. Contudo, pode ser observado, apesar do grau de conservação, que o fragmento apresenta indícios de corte seletivo de madeira. 
O solo predominante na área I é o ARGISSOLO VERMELHO-AMARELO Eutrófico abrúptico, textura média/argilosa e o mesmo tipo de solo é encontrado na área II, com pequenas variações, além de um VERMELHO-AMARELO Distrófico Plíntico ou nãoPlíntico, textura média/argilosa (Santos et al., 2013).

\section{Coleta e tratamento de dados}

O levantamento fitossociológico foi realizado adotando-se o método de parcelas, proposto por MuellerDombois \& Ellemberg (1974). Foram estabelecidas 10

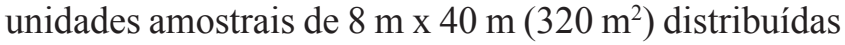
de maneira aleatória em ambas as áreas (Calixto Júnior et al. 2011). Em cada unidade de parcela foram contabilizados todos os indivíduos vivos, com diâmetro ao nível do solo (DNS) igual ou superior a $3 \mathrm{~cm}$ (Queiroz et al., 2006) e medida a altura total de cada árvore, utilizando-se suta dendrométrica e vara telescópica graduada, respectivamente.

A identificação foi realizada primeiramente em campo, com ajuda de mateiro e depois, através de morfologia comparada, usando bibliografia especializada e análise das exsicatas depositadas no Herbário do Trópico Semiárido (HTSA) da Embrapa Semiárido. As espécies foram classificadas nas famílias reconhecidas pelo APG III (Angiosperm Phylogeny Group, 2009) e a lista dos autores das espécies foi organizada segundo Brummitt \& Powell (1992). A análise fitossociológica foi realizada com o auxílio do software Mata Nativa versão 2.04 (Cientec, 2002), que possibilitou a análise dos parâmetros gerais da comunidade (densidade total, área basal total, alturas e diâmetros) e parâmetros relativos das espécies (DA - densidade absoluta; DR - densidade relativa; FA - frequência absoluta; FR - frequência relativa; DoA - dominância absoluta; DoR - dominância relativa e IVI - índice de valor de importância) conforme Mueller-Dombois \& Ellemberg (1974).

Para verificar o padrão de distribuição espacial das espécies nas áreas I e II utilizou-se o índice de agregação de Payandeh - Pi (Payandeh, 1970).

Com o objetivo de identificar a similaridade florística das áreas com outros levantamentos realizados em áreas de Caatinga foi elaborada uma matriz de presença/ ausência de espécies citadas em outros 14 levantamentos: (Drumond et al., 2002; Alcoforado-Filho et al., 2003; Maracajá et al,. 2003; Amorim et al., 2005; Silva, 2005; Andrade et al., 2005; Queiroz et al., 2006; Santana \& Souto, 2006; Araújo, 2007; Fabricante \& Andrade, 2007; Cavalcanti, 2008; Costa, 2008; Pessoa et al., 2008).
Em seguida foram construídos dendrogramas para os dados referentes à vegetação existente nas áreas a serem comparadas, utilizando-se a distância euclidiana como medida de dissimilaridade e o algoritmo de agrupamento de Ward, através do software SAEG 5.0 (Sistema..., 1997). Através do software PC-Ord (Mccune, 1999), outra análise de agrupamento foi conduzida, utilizandose o índice de Jaccard e o parâmetro fitossociológico DA (ind.ha ${ }^{-1}$ ) de cada comunidade ou levantamento, tomando-se a mesma medida de dissimilaridade (distância euclidiana) e o mesmo algoritmo (método de Ward). Comparações entre as médias dos parâmetros da estrutura horizontal (AB e DA) e entre os valores dos índices de diversidade de Shannon e equabilidade de Pielou foram feitas utilizando-se o teste $\mathrm{t}$ de Student, a $1 \%$ e $5 \%$ de probabilidade.

\section{Resultados e discussão}

\section{Florística}

Na área I foram inventariados 432 indivíduos, distribuídos em 07 famílias, 14 gêneros e 16 espécies, enquanto que na área II foram amostrados 296 indivíduos pertencentes a 09 famílias, 21 gêneros e 25 espécies. Foram observadas 14 espécies comuns às duas áreas (Tabela 1 ).

As famílias mais importantes tanto em número de espécies quanto de indivíduos em ambas as áreas foram as que aparecem com destaque, dentre as mais representativas, na grande maioria dos estudos florísticos em caatinga: Fabaceae e Euphorbiaceae. Estas famílias abrangem a maior parte das espécies lenhosas da flora do bioma, corroborando com Andrade et al., (2007) e outros (Pereira et al., 2003; Alcoforado-Filho et al., 2003; Andrade et al. 2005; Silva, 2005; Rodal et al., 2008), inclusive com resultados de levantamentos realizados no Município de Petrolina, PE (Drumond et al., 2002; Nascimento et al., 2003; Fabricante, 2007). Pôde-se observar uma maior diversidade florística na área melhor conservada (área II) em relação à área que sofreu corte raso há três décadas (área I). Estes valores, revelados pelo índice de diversidade de Shannon $\left(H^{\prime}\right)$ para as áreas I e II, respectivamente, foram 1,39 e 2,52 (diferenças estatísticas significativas para o teste $\mathrm{t}<$ $0,05)$, estando de acordo outros estudos realizados em ambientes de Caatinga, que variam de 1,10 a 3,09 nats ind $^{-1}$. Esses resultados são semelhantes aos encontrados por Andrade et al. (2005), que ao estudarem duas fitofisionomias de Caatinga com diferentes históricos de 
uso em São João do Cariri, PB, observaram que a área com histórico de intensa antropização, também com 30 anos de regeneração, apresentou apenas 5 famílias e 6 espécies. $\mathrm{Na}$ área em melhor estado de conservação os autores observaram maior número de famílias e espécies, corroborando com os resultados obtidos por Pereira et al. (2003), que verificaram a ocorrência de apenas 10 espécies e 6 famílias em uma área usada para agricultura seguido de pastagem durante 50 anos, com 20 anos de regeneração no Agreste Paraibano e por Luna (1998), que observou em duas áreas degradadas em São João do Cariri, $\mathrm{PB}$, a presença de 08 farmílias e 10 espécies (área A) e 06 famílias e 08 espécies (área B).

A homogeneidade na distribuição das espécies (demonstrada de forma mais expressiva na área I) é resultante de ações antrópicas ocorridas nos fragmentos no passado. A similaridade entre as áreas, estimada pelo índice de Jaccard, foi igual a 0,52, revelando alta homogeneidade florística, fato decorrente da proximidade geográfica entre os fragmentos.

Tabela 1. Listagem das espécies inventariadas nos dois fragmentos de Caatinga, Embrapa Semiárido, Petrolina, PE. Em que: ( - ) ausente e ( $\mathrm{X}$ ) presente.

\begin{tabular}{|c|c|c|c|c|}
\hline \multirow{2}{*}{ Família } & \multirow{2}{*}{ Espécie } & \multirow{2}{*}{ Nome Comum } & \multicolumn{2}{|c|}{ Área } \\
\hline & & & I & II \\
\hline \multirow[t]{3}{*}{ Anacardiaceae } & Myracrodruon urundeuva Allemão & Aroeira & - & $\mathrm{X}$ \\
\hline & Schinopsis brasiliensis Engl. & Baraúna & $\mathrm{X}$ & $\mathrm{X}$ \\
\hline & Spondias tuberosa Arruda & Umbuzeiro & - & $\mathrm{X}$ \\
\hline Apocynaceae & Aspidosperma pyrifolium Mart. & Pereiro & $\mathrm{X}$ & $\mathrm{X}$ \\
\hline Bignoniaceae & Tabebuia spongiosa Rizzini & Sete cascas & $\mathrm{X}$ & $\mathrm{X}$ \\
\hline Burseraceae & Commiphora leptophloeos (Mart.) J.B. Gillett & Umburana de cambão & - & $\mathrm{X}$ \\
\hline Cactaceae & Cereus jamacaru DC. & Mandacaru de boi & $\mathrm{X}$ & $\mathrm{X}$ \\
\hline \multirow[t]{7}{*}{ Euphorbiaceae } & Croton conduplicatus Kunth & Quebra faca & - & $\mathrm{X}$ \\
\hline & Croton sonderianus Müll. Arg. & Marmeleiro preto & - & $\mathrm{X}$ \\
\hline & Cnidoscolus quercifolius Pohl. & Favela comum & $\mathrm{X}$ & $\mathrm{X}$ \\
\hline & Cnidoscolus bahianus (Ule) Pax \& K. Hoffm. & Favela de galinha & - & $\mathrm{X}$ \\
\hline & Manihot pseudoglaziovii Pax \& K. Hoffm. & Maniçoba & $\mathrm{X}$ & $\mathrm{X}$ \\
\hline & Jatropha mollissima (Pohl) Baill. & Pinhão vermelho & $\mathrm{X}$ & $\mathrm{X}$ \\
\hline & Sapium lanceolatum (Müll. Arg.) Huber & Burra leiteira & - & $\mathrm{X}$ \\
\hline \multirow[t]{10}{*}{ Fabaceae } & Anadenanthera colubrina (Vell.) Brenan & Angico vermelho & $\mathrm{X}$ & $\mathrm{X}$ \\
\hline & Bauhinia cheilantha (Bong.) Steud. & Mororó & $\mathrm{X}$ & - \\
\hline & Caesalpinia ferrea Mart. ex Tul. & Jucá & $\mathrm{X}$ & - \\
\hline & Caesalpinia microphylla Mart. ex G. Don & Catingueira rasteira & $\mathrm{X}$ & $\mathrm{X}$ \\
\hline & Chloroleucon margense (Jacq.) Britton \& Rose & Arapiraca & - & $\mathrm{X}$ \\
\hline & Mimosa arenosa (Willd.) Poir. & Jurema vermelha & $\mathrm{X}$ & $\mathrm{X}$ \\
\hline & Mimosa tenuiflora (Willd.) Poir. & Jurema preta & $\mathrm{X}$ & $\mathrm{X}$ \\
\hline & Piptadenia stipulacea (Benth.) Ducke & Unha de gato & $\mathrm{X}$ & $\mathrm{X}$ \\
\hline & Pithecellobium diversifolium Benth. & Espinheiro & - & $\mathrm{X}$ \\
\hline & $\begin{array}{l}\text { Senna macranthera (DC. ex Collad.) } \\
\text { H.S. Irwin \& Barneby }\end{array}$ & São João & $\mathrm{X}$ & $\mathrm{X}$ \\
\hline Malvaceae & Pseudobombax simplicifolium A. Robyns & Imbiruçu & - & $\mathrm{X}$ \\
\hline Verbenaceae & Lippia microphylla Cham. & Alecrim & $\mathrm{X}$ & $\mathrm{X}$ \\
\hline
\end{tabular}


Os valores do índice de Equabilidade de Pielou $(J)$ foram 0,50 e 0,78 , respectivamente (diferenças estatísticas significativas para o Teste $t<0,05$ ), indicando que na área II não houve predomínio acentuado de uma ou de poucas espécies sobre as outras, diferentemente da área I, na qual a baixa equabilidade foi fortemente influenciada pela alta densidade de $M$. tenuiflora, que se fez presente em todas as parcelas, apontando heterogeneidade florística baixa na área. Infere-se a isto, o fato de $M$. tenuiflora formar matas quase puras no início da sucessão, por se tratar de espécie colonizadora, apontando o estágio progressivo de sucessão secundária a qual a área I se encontra.

Os táxons mais abundantes na área I foram: Mimosa tenuiflora, Mimosa arenosa e Tabebuia spongiosa, sendo estes responsáveis por $81,2 \%$ do total amostrado. $\mathrm{Na}$ área II Mimosa tenuiflora foi a espécie com maior densidade, seguida de Caesalpinia microphylla e Manihot pseudoglaziovii, sendo estes responsáveis por $49,6 \%$ do total de indivíduos inventariados.

$\mathrm{O}$ valor de área basal $(\mathrm{AB})$ na área I correspondeu a $7,28 \mathrm{~m}^{2}$.ha ${ }^{-1}$, sendo considerado baixo, porém de acordo com outros valores encontrados em ambientes de Caatinga, que variam de 4 a $52 \mathrm{~m}^{2} \cdot \mathrm{ha}^{-1}$ (Sampaio, 1996). Na área II o valor de $\mathrm{AB}$ foi de $40,23 \mathrm{~m}^{2}$.ha-1, sendo superior ao encontrado por Alcoforado-Filho (2003) $\left(24,9 \mathrm{~m}^{2} . \mathrm{ha}^{-1}\right) \mathrm{e}$ Santana \& Souto (2006) $\left(17,50 \mathrm{~m}^{2} \cdot \mathrm{ha}^{-1}\right)$. A densidade total foi de 1.350 ind.ha $^{-1}$ na área I, sendo inferior à grande parte dos trabalhos em Caatinga, que variam de 1.437 a 3.576 ind. ha $^{-1}$ (Drumond et al., 2002; Alcoforado-Filho et al., 2003; Andrade et al., 2005; Fabricante, 2007; Rodal et al., 2008), o que se explica pela grande variabilidade das fisionomias de Caatinga ou por diferenças nas pressões antrópicas exercidas por diferentes tipos de usos. A área II apresentou densidade menor (925 ind.ha ${ }^{-1}$ ), fato explicado pela presença marcante de regenerantes com valores de DNS menores que $3 \mathrm{~cm}$. Diferenças estatísticas significativas foram encontradas, ao considerar os valores de $\mathrm{AB}$ e $\mathrm{DA}$ para ambas as áreas de estudo (teste t, $P<0,01$ ). Os valores relativos dos parâmetros estruturais das comunidades estão representados na Tabela 3.

A distribuição dos indivíduos em classes diamétricas da área I revela a existência de representantes em todas as classes estabelecidas. Verificou-se que os maiores números de indivíduos ocorreram nas três primeiras classes $(91,19 \%)$ : de 3 a $6 \mathrm{~cm}(36,80 \%)$, de 6,1 a 9 $\mathrm{cm}(32,40 \%)$ e de 9,1 a $12 \mathrm{~cm}(21,99 \%)$, enquanto nas sete classes de maior diâmetro, só foram observados 38 indivíduos (8,79\%). Essa distribuição se aproxima do modelo de distribuição exponencial na forma de "J invertido", padrão característico de florestas inequiâneas. Aparentemente, esse padrão se deveu basicamente ao comportamento das duas espécies com maior valor de importância (M. tenuiflora e M. arenosa), ambas com elevado número de indivíduos nas classes de menor diâmetro. $\mathrm{O}$ maior diâmetro observado na área I foi de $30,7 \mathrm{~cm}$ pertencente a um indivíduo de C. quercifolius e o diâmetro médio foi de $6,7 \mathrm{~cm}$, sendo considerado pequeno e fortemente afetado pela presença de muitas espécies arbustivas, as quais normalmente possuem caules de pequeno diâmetro. Na área II verificou-se que os maiores números de indivíduos não ocorrem na primeira, mas entre a segunda e a quarta classes, de 6,1 a $9 \mathrm{~cm}(13,51 \%), 9,1$ a $12 \mathrm{~cm}(13,17 \%)$ e 12,1 a 15 $\mathrm{cm}(15,87 \%)$, totalizando $48 \%$ do total de indivíduos. O maior diâmetro observado foi de 50,2 cm (Figura 1), pertencente a um indivíduo de C. leptopholeos. Foi observada, mais uma vez, a tendência das florestas secundárias, ou seja, elevada proporção de indivíduos regenerantes em relação aos adultos, apesar da área II apresentar menor número de indivíduos estoque e maior número de árvores inseridas em classes de maior diâmetro, sugerindo antropização menos intensa ou menos recente que a área $\mathrm{I}$.

Almeida Neto et al. (2009), em estudo no semiárido paraibano e Marangon et al. (2013), estudando área de caatinga em Floresta, PE, obtiveram distribuição diamétrica representada na forma de $\mathrm{J}$ invertido. Os primeiros autores observaram 2.690 indivíduos distribuídos nas três primeiras classes e 1.975 distribuídos somente na primeira classe, com queda acentuada para as classes seguintes, corroborando com os resultados deste trabalho (área I). Marangon et al. (2013) apontam 701 indivíduos na primeira classe e 325 na segunda, contribuindo com $83 \%$ dos indivíduos amostrados em toda a área de estudo.

A distribuição hipsométrica dos indivíduos da área I mostra configuração com maior concentração de plantas nas classes mais inferiores, com a maioria dos indivíduos agrupados na segunda classe $(3,1 \mathrm{a} 4 \mathrm{~m})$ representando um total de $58,8 \%$ dos indivíduos inventariados. Notouse a ocorrência de gradual redução à medida que se aproxima das classes com maior valor de altura. A maior altura registrada foi de 7,9 m para um indivíduo de $M$. arenosa. Entre a primeira e segunda, e a segunda e a terceira classes, observam-se um aumento e uma queda abrupta, respectivamente, no número de indivíduos, explicada pela intensa intervenção no processo natural de sucessão, mais necessariamente, provocado pelo corte raso realizado no fragmento por volta do ano de 1979. 
Tabela 2. Parâmetros fitossociológicos das espécies amostradas em dois fragmentos de caatinga em Petrolina, PE.

\begin{tabular}{|c|c|c|c|c|c|c|c|c|c|c|}
\hline \multirow{2}{*}{ Espécies } & \multicolumn{6}{|c|}{ Área I } & \multicolumn{4}{|c|}{ Área II } \\
\hline & $\mathbf{N}$ & DR & FR & DoR & VI & $\mathbf{N}$ & DR & FR & DoR & VI \\
\hline M. tenuiflora & 283 & 65,51 & 14,49 & 69,6 & 149,59 & 61 & 20,61 & 9,30 & 26,8 & 56,70 \\
\hline M. arenosa & 42 & 9,72 & 11,59 & 7,69 & 29,00 & 14 & 4,73 & 6,98 & 5,9 & 17,60 \\
\hline M. pseudoglaziovii & 18 & 4,17 & 13,04 & 4,47 & 21,68 & 29 & 9,8 & 8,14 & 3,11 & 21,04 \\
\hline T. spongiosa & 26 & 6,02 & 11,59 & 2,79 & 20,40 & 17 & 5,74 & 4,65 & 9,17 & 19,56 \\
\hline C. quercifolius & 15 & 3,47 & 8,70 & 6,40 & 18,57 & 17 & 5,74 & 8,14 & 3,51 & 17,54 \\
\hline B. cheilantha & 14 & 3,24 & 10,14 & 0,87 & 14,25 & 0 & 0.00 & 0.00 & 0.00 & 0.00 \\
\hline M. stipulaceae & 7 & 1,62 & 5,80 & 3,02 & 10,43 & 1 & 0,34 & 1,16 & 0,09 & 1,59 \\
\hline J. molissima & 8 & 1,85 & 7,25 & 0,62 & 9,72 & 16 & 5,41 & 8,14 & 0,84 & 14,38 \\
\hline L. microphylla & 7 & 1,62 & 5,80 & 0,70 & 8,12 & 4 & 1,35 & 2,33 & 0,2 & 3,87 \\
\hline A. pyrifolium & 5 & 1,16 & 2,90 & 0,94 & 4,99 & 11 & 3,72 & 3,49 & 5,82 & 13,02 \\
\hline A. colubrina & 2 & 0,46 & 1,45 & 1,54 & 3,45 & 1 & 0,34 & 1,16 & 0,13 & 1,63 \\
\hline C. jamacaru & 1 & 0,23 & 1,45 & 0,54 & 2,21 & 2 & 0,68 & 2,33 & 1,13 & 4,13 \\
\hline C. microphylla & 1 & 0,23 & 1,45 & 0,51 & 2,19 & 57 & 19,26 & 8,14 & 8,23 & 35,62 \\
\hline S. macranthera & 1 & 0,23 & 1,45 & 0,18 & 1,85 & 1 & 0,34 & 1,16 & 0,05 & 1,54 \\
\hline C. férrea & 1 & 0,23 & 1,45 & 0,07 & 1,74 & 0 & 0.00 & 0.00 & 0.00 & 0.00 \\
\hline S. brasiliensis & 1 & 0,23 & 1,45 & 0,06 & 1,74 & 3 & 1,01 & 2,33 & 0,24 & 3,57 \\
\hline M. urundeuva & 0 & 0,00 & 0,00 & 0,00 & 0,00 & 4 & 1,35 & 2,33 & 4,68 & 8,35 \\
\hline C. leptophloeos & 0 & 0,00 & 0,00 & 0,00 & 0,00 & 10 & 3,38 & 5,81 & 12,99 & 22,18 \\
\hline C. bahiensis & 0 & 0,00 & 0,00 & 0,00 & 0,00 & 14 & 4,73 & 8,14 & 7,20 & 20,06 \\
\hline C. conduplicatus & 0 & 0,00 & 0,00 & 0,00 & 0,00 & 24 & 8,11 & 6,98 & 2,46 & 17,54 \\
\hline S. tuberosa & 0 & 0,00 & 0,00 & 0,00 & 0,00 & 1 & 0,34 & 1,16 & 3,71 & 5,20 \\
\hline S. lanceolatum & 0 & 0,00 & 0,00 & 0,00 & 0,00 & 3 & 1,01 & 2,33 & 1,05 & 4,38 \\
\hline P. simplicifolium & 0 & 0,00 & 0,00 & 0,00 & 0,00 & 1 & 0,34 & 1,16 & 1,99 & 3,49 \\
\hline F. multiflora & 0 & 0,00 & 0,00 & 0,00 & 0,00 & 2 & 0,68 & 1,16 & 0,42 & 2,26 \\
\hline P. diversifolium & 0 & 0,00 & 0,00 & 0,00 & 0,00 & 1 & 0,34 & 1,16 & 0,18 & 1,68 \\
\hline C. margense & 0 & 0,00 & 0,00 & 0,00 & 0,00 & 1 & 0,34 & 1,16 & 0,09 & 1,59 \\
\hline C. sonderianus & 0 & 0,00 & 0,00 & 0,00 & 0,00 & 1 & 0,34 & 1,16 & 0,02 & 1,52 \\
\hline
\end{tabular}

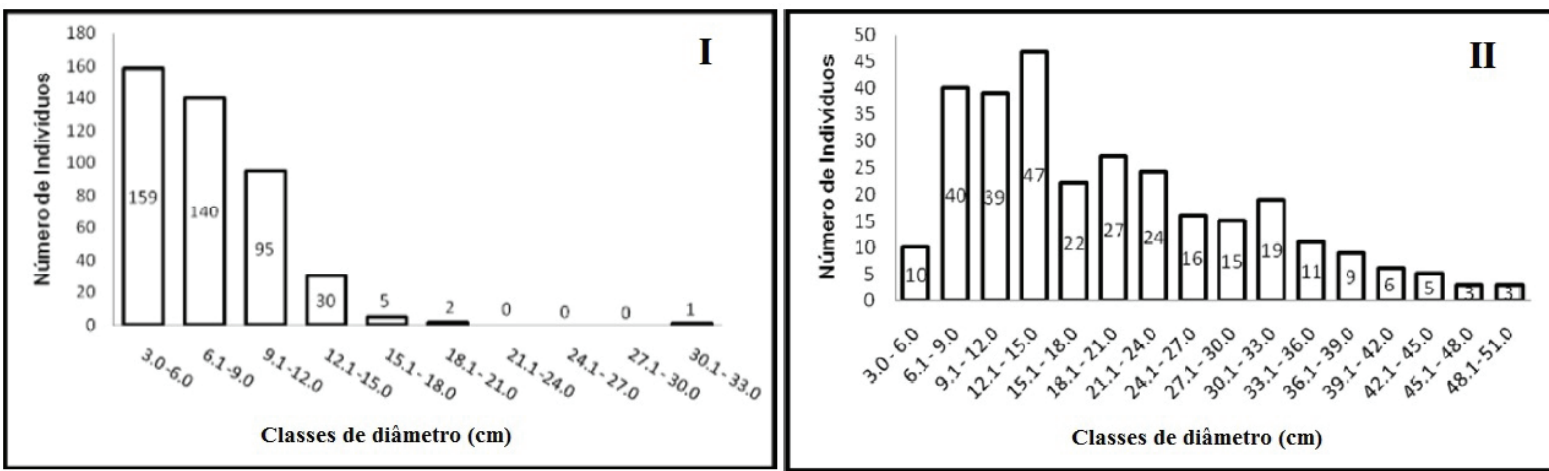

Figura 1. Distribuição do número de indivíduos por classe de diâmetro com amplitude de $3 \mathrm{~cm}$, áreas I e II, Embrapa Semiárido, Petrolina, PE. 
De acordo com o porte médio obtido para as formas de vida na área I, e seguindo a classificação de MullerDombois \& Ellemberg (1974), 28 plantas (6,7\%) podem ser consideradas como nanofanerófitas (1 - 2 $\mathrm{m})$, mais precisamente com $2 \mathrm{~m}$ de altura e $93,3 \%$ como microfanerófitas $(2-8 \mathrm{~m})$, podendo-se assim caracterizar a vegetação da área I como sendo do tipo arbóreo-arbustiva.

De um modo geral, observou-se que o fragmento em questão apresenta a maioria de seus indivíduos de pequeno porte, ou seja, com altura inferior a $5 \mathrm{~m}$, inferindo-se que se apresenta em estágio inicial de sucessão.

$\mathrm{Na}$ área II, em relação à distribuição vertical, observou-se que $81,08 \%$ de seus indivíduos (240) estão agrupados nas três primeiras classes hipsométricas (2-5 m). Valores semelhantes foram encontrados por Sampaio \& Rodal (2000), Alcoforado-Filho et al. (2003) e Almeida-Neto (2008) em áreas também consideradas bem conservadas. As maiores alturas foram de $8,2 \mathrm{~m}$ para um individuo de $M$. urundeuva e 7,6 m para um de C. quercifolius. Observou-se também que o fragmento apresenta a maioria de seus indivíduos de pequeno porte (altura inferior a $5 \mathrm{~m}$ ), apesar de possuir um número maior de indivíduos arbóreos representados por classes de altura maiores.

\section{Agregação das espécies}

O padrão de distribuição espacial na área I, medido pelo índice de Payandeh (Payandeh, 1970), apresentou 2 táxons $(12,5 \%)$ (M. pseudoglaziovii e $B$. cheilantha) com tendência ao agrupamento, $5(31,25 \%)(C$. jamacaru, $C$. microphylla, $S$. macranthera, C. ferrea e $S$. brasiliensis) com padrão não agrupamento (aleatórias) e 9 (56,25\%) com padrão agrupamento (uniformes). Na área II apenas 1 táxon (4\%) (Jatropha molissima) apresentou tendência ao agrupamento, enquanto 9 (36\%) (M. tenuiflora, C. microphylla, C. leptophloeos, M. glaziovii, C. bahianus, Tabebuia spongiosa, Mimosa arenosa, Croton conduplicatus e Cnidoscolus quercifolius) apresentaram padrão de aleatórias, as demais espécies $(15-60 \%)$ apresentaram padrão uniforme (Figura 2).

Observa-se que as áreas I e II apresentaram comportamentos semelhantes, ou seja, o maior número de espécies $(56,25 \%$ e $60 \%$ respectivamente) apresentando padrão uniforme (agrupamento), seguidos de padrão aleatório ( $31,25 \%$ e $36 \%$ respectivamente) e tendência a agrupamento $(12,5 \%$ e $4 \%$ respectivamente).

Esses resultados corroboram com os obtidos por Santana (2009) e e Mendes Júnior et al. (2009), que mostram ser comum a ocorrência deste fato em ambientes de caatinga, onde espécies mais abundantes surgem agrupadas ou com tendência ao agrupamento. Para Mendes Júnior et al. (2009), isso se deve a uma estratégia da Caatinga de se estabelecer com grande quantidade de indivíduos em áreas que sofreram alguma perturbação.

Neste trabalho, entende-se este padrão de distribuição das espécies em sua maioria, como influência de fatores biológicos das espécies, como a dispersão a curta distância e fatores ambientais, como solo raso e distúrbios naturais.

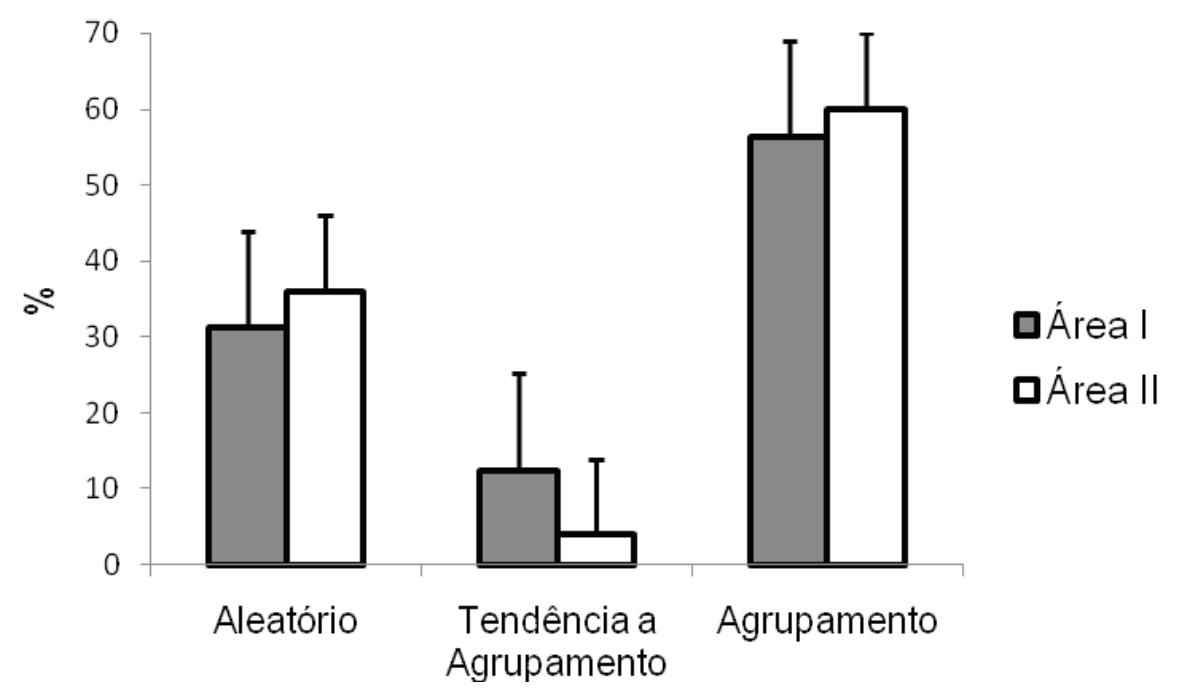

Figura 2. Proporção dos diferentes padrões de distribuição espacial nas áreas I e II, Embrapa Semiárido, Petrolina, PE. 


\section{Similaridade}

Através da análise de similaridade florística entre as áreas estudadas e outros 14 levantamentos realizados em ambientes de caatinga sensu stricto nos estados do
Pernambuco, Paraíba e Rio Grande do Norte (Tabela 3), foi possível observar a formação de 5 grupos em nível de distância euclidiana máxima (100\%).

Tabela 3. Listagem das 16 áreas utilizadas na análise de agrupamento representadas pelos códigos (A a P). Onde: NE = número de espécies; NF = Número de Famílias; DA = Densidade absoluta.

\begin{tabular}{cccccccc}
\hline Resultado & NE & NF & $\begin{array}{c}\text { Área } \\
(\mathbf{h a})\end{array}$ & $\begin{array}{c}\text { Precipitação } \\
\left(\mathbf{m m} \mathbf{a n o}^{-1}\right)\end{array}$ & $\begin{array}{c}\text { Altura } \\
(\mathbf{m})\end{array}$ & $\begin{array}{c}\text { Critério de } \\
\text { inclusã } \mathbf{0}^{*}\end{array}$ & DA (ind/ha $\left.{ }^{-1}\right)$ \\
\hline $\mathbf{A}$ & 16 & 8 & 0,32 & 535 & 382 & DNS $\geq 3 \mathrm{~cm}$ & 1.350 \\
$\mathbf{B}$ & 25 & 11 & 0,32 & 535 & 382 & $\mathrm{DNS} \geq 3 \mathrm{~cm}$ & 925 \\
$\mathbf{C}$ & 21 & 9 & 2 & 535 & 350 & $\mathrm{DAP} \geq 5 \mathrm{~cm}$ & 362 \\
$\mathbf{D}$ & 28 & 11 & 1 & 511 & 545 & $\mathrm{DNS} \geq 3 \mathrm{~cm}$ & 3.567 \\
$\mathbf{E}$ & 22 & 14 & 2 & 758 & 250 & $\mathrm{CAS} \geq 10 \mathrm{~cm}$ & 1.437 \\
$\mathbf{F}$ & 32 & 19 & 2 & 801 & 250 & $\mathrm{CAS} \geq 10 \mathrm{~cm}$ & 2.220 \\
$\mathbf{G}$ & 22 & 8 & 0,40 & 569 & 271 & $\mathrm{DNS} \geq 3 \mathrm{~cm}$ & 3.130 \\
$\mathbf{H}$ & 15 & 10 & 1 & 758 & 250 & $\mathrm{CAP} \geq 3 \mathrm{~cm}$ & 3.250 \\
$\mathbf{I}$ & 22 & 12 & 0,60 & 758 & 250 & $\mathrm{DNS} \geq 3 \mathrm{~cm}$ & 3.372 \\
$\mathbf{J}$ & 14 & 9 & 0,24 & 600 & 215 & $\mathrm{CAB} \geq 10 \mathrm{~cm}$ & 687 \\
$\mathbf{K}$ & 10 & 6 & 0,68 & 715 & 650 & $\mathrm{CAP} \geq 6 \mathrm{~cm}$ & 1.941 \\
$\mathbf{L}$ & 15 & 8 & 0,24 & 386 & 500 & $\mathrm{CAB} \geq 10 \mathrm{~cm}$ & 1.471 \\
$\mathbf{M}$ & 27 & 15 & 0,96 & 747 & 250 & $\mathrm{CAP} \geq 10 \mathrm{~cm}$ & 1.775 \\
$\mathbf{N}$ & 8 & 5 & 0,24 & 750 & 130 & $\mathrm{CAB} \geq 10 \mathrm{~cm}$ & 538 \\
$\mathbf{O}$ & 22 & 12 & 0,72 & 694 & 530 & DAS $\geq 3 \mathrm{~cm}$ & 3.810 \\
$\mathbf{P}$ & 28 & 16 & 0,20 & 450 & 355 & DNS $\geq 3 \mathrm{~cm}$ & 460 \\
\hline
\end{tabular}

*DNS (diâmetro ao nível do solo); DAP (diâmetro à altura de peito); CAS (circunferência à altura do solo); CAP (circunferência à altura do peito); CAB (circunferência à altura da base); DAS (diâmetro à altura do solo). Sendo: (A) Área I - Este trabalho - Petrolina, PE; (B) Área II - Este trabalho - Petrolina, PE; (C) Drumond et al. (2002) - Petrolina, PE; (D) Cavalcanti (2008) - Betânia, PE; (E) Silva (2005) - Serra Negra do Norte, RN; (F) Silva (2005) - Condado, PB; (G) Fabricante \& Andrade (2007) - Santa Luzia, PB; (H) Amorim et al. (2005) - Serra Negra do Norte, RN; (I) Santana \& Souto (2006) - Serra Negra do Norte, RN; (J) Maracajá et al. (2003) - Ambiente I, Serra do Mel, RN; (K) Costa (2008) - São José do Bomfim, PB (Dados não publicados); (L) Andrade et al. (2005) - São João do Cariri, PB; (M) Araújo (2007) - Santa Terezinha, PB; (N) Pessoa et al. (2008) - Apodi, RN; (O) Alcoforado-Filho et al. (2003) Caruaru, PE; (P) Queiroz, et al. (2006) - Boqueirão, PB.

O primeiro grupo, composto pelos trabalhos A, B e $\mathrm{C}$, foi formado pelos levantamentos realizados em Petrolina, PE. O segundo grupo agrega os levantamentos realizados em Serra Negra do Norte, RN (E), Santa Terezinha, PB (M) e Condado, PB (F). O terceiro grupo, formado pelos levantamentos $\mathrm{O}$ e $\mathrm{P}$, foram realizados em Caruaru, $\mathrm{PE}$ e Boqueirão, $\mathrm{PB}$, respectivamente. O quarto grupo une os levantamentos realizados em Santa Luzia, PB (G), Apodi, RN (N) e Serra do Mel, RN (J) e o quinto grupo relaciona os trabalhos $\mathrm{K}, \mathrm{L}, \mathrm{D}, \mathrm{H}$ e I realizados em São José do Bomfim, PB, São João do Cariri, PB, Betânia, PE e os dois últimos em Serra Negra do Norte, $\mathrm{RN}$, respectivamente (Figura 3).

O principal fator responsável pela similaridade florística revelada na análise de agrupamento foi, possivelmente, a proximidade geográfica entre as áreas, aliada aos atuais estados de conservação e históricos de uso das mesmas, que também pode ser comprovado pelos valores do índice de Jaccard obtidos na comparação dos dados de cada área. Além disso, observa-se que cada grupo, ou fragmento isolado, apresenta um conjunto de características peculiares (altitude, tipologia de solos, pluviometria), o que ressalta a sua importância quanto à conservação e manejo de sua flora.

Outra análise de agrupamento foi conduzida, utilizando-se a mesma medida de dissimilaridade (distância euclidiana) e o mesmo algoritmo (método de Ward), porém, utilizando-se o parâmetro fitossociológico DA (ind. ha ${ }^{-1}$ ) de cada comunidade ou levantamento. Com os novos dados na matriz, foi gerado o dendrograma correspondente (Figura 4). 


\section{Distância Euclidiana Simples}

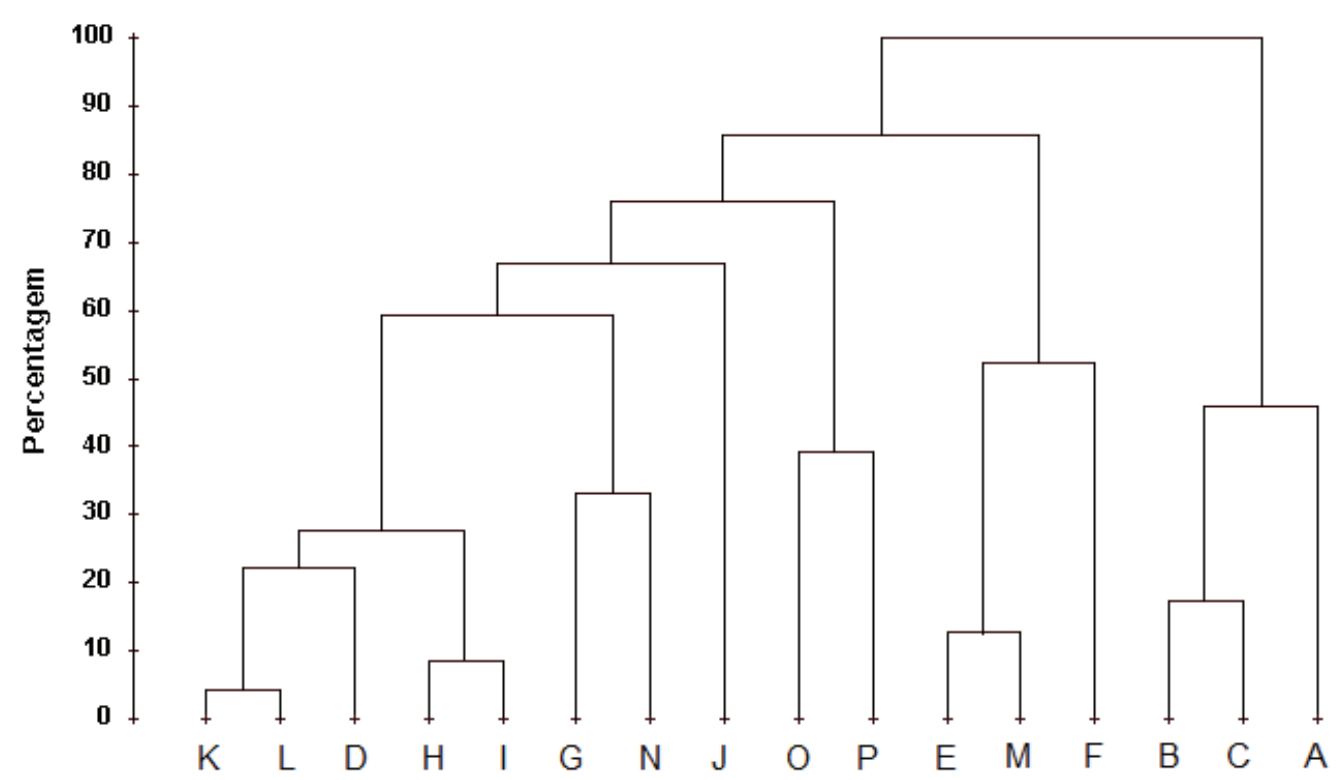

Figura 3. Dendrograma gerado pelo método de Ward, distância euclidiana, com as variáveis padronizadas da matriz de presença e ausência de espécies para os 16 levantamentos nos estados de Pernambuco, Paraíba e Rio Grande do Norte.

\section{Distância Euclidiana}

\section{Distance (Objective Function)}
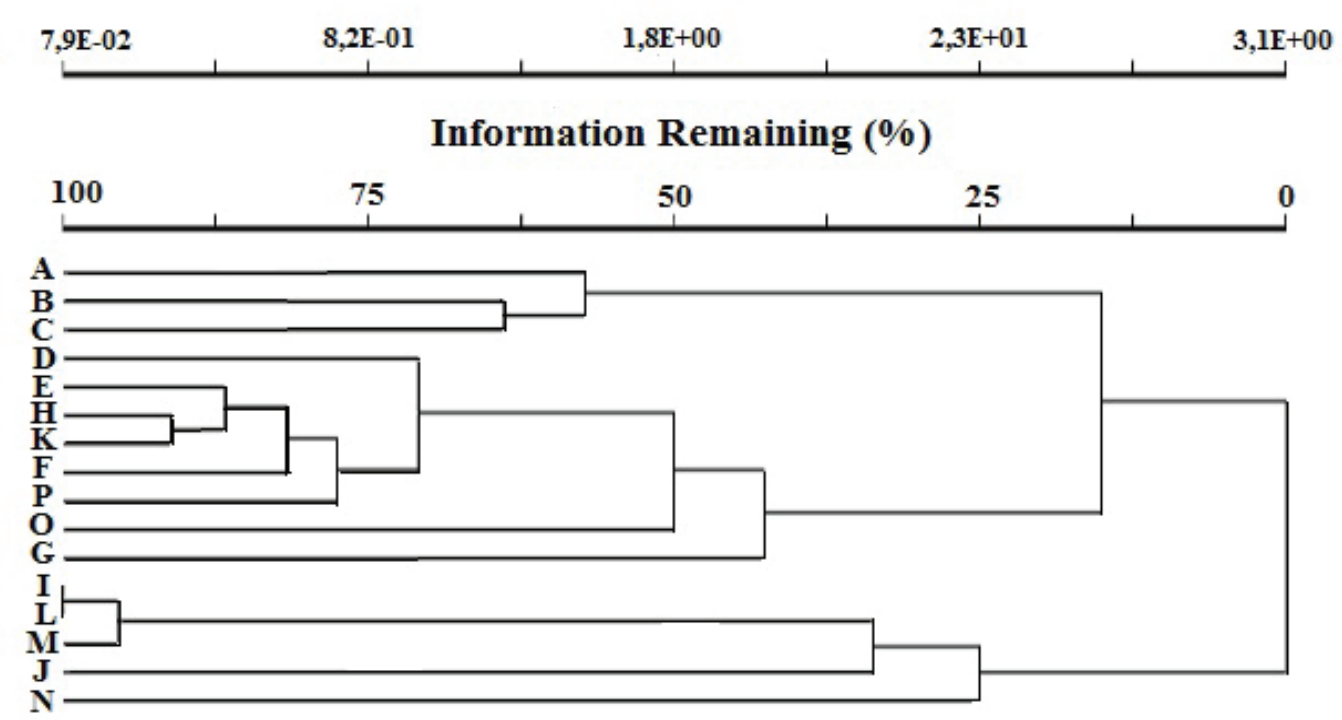

Figura 4. Dendrograma obtido pelo método de Ward, distância euclidiana, com o parâmetro de estrutura horizontal DA das espécies encontradas nos 16 levantamentos nos estados de Pernambuco, Paraíba e Rio Grande do Norte. 
$\mathrm{Na}$ Figura 4 é apresentada a formação de três agrupamentos, um formado pelas áreas A, B e C, outro formado por D, E, H, K, F, P, O e G e um último formado pelas áreas I, L, M, J e N.

É possível observar que as áreas de Caatinga sensu stricto postas em comparação apresentam elementos florísticos que se distinguem por vários fatores em diferentes escalas, desta forma, não apresentam grande quantidade de espécies em comum. Os agrupamentos, entretanto, foram formados pela singularidade existente entre as áreas no que se refere também à densidade absoluta das espécies. Drumond et al. (2002) observaram em uma área de Caatinga na Embrapa Semiárido em Petrolina, que as espécies $M$. tenuiflora, C. mycrophylla e $C$. phyllacanthus foram também as mais abundantes. Segundo Rodal et al. (2008) para saber se as formações de caatinga arbustivo-arbóreas resultam de habitats particulares ou estão ligadas a questões dos estádios sucessionais é necessário que haja um maior número de levantamentos englobando áreas onde o conhecimento do histórico de uso seja inequívoco, as condições de habitats sejam similares e se possível tenham formato, tamanho e matriz circundante equivalentes.

\section{Conclusões}

O elevado número de indivíduos com diâmetro e altura reduzidos no fragmento que sofreu corte raso há três décadas (área I), aponta a existência de uma comunidade ainda em fase de recuperação da estrutura original, em estágio inicial de sucessão, e mesmo as áreas estando próximas uma da outra, foi possível observar diferenças tanto fisionômicas, quanto estruturais, evidenciando que este fragmento ainda não alcançou um estágio de desenvolvimento ecológico semelhante à área em melhor estágio de conservação (área II).

$\mathrm{O}$ corte raso realizado em ambiente de caatinga provocou diminuição da diversidade, dominância, diâmetro e altura das plantas e o intervalo de 30 anos não foi suficiente para a comunidade vegetal atingir um estágio avançado de desenvolvimento ecológico.

\section{Referências}

ALCOFORADO FILHO, F. G. A.; SAMPAIO, E. V. B.; RODAL, M. J. N. Florística e fitossociologia de um remanescente de vegetação caducifólia espinhosa arbórea em Caruaru, Pernambuco. Acta Botânica Brasílica, São Paulo, v. 17, n. 2, p. 287-303, 2003.
ALMEIDA NETO, J. X.; ANDRADE, A. P.; LACERDA, A. V.; FELIX, L. P.; BRUNO, R. L. A. Composição florística, estrutura e análise populacional do feijão-bravo (Capparis flexuosa L.) no semiárido paraibano, Brasil. Revista Caatinga, Mossoró, v. 22, n. 4, p. 187-194, 2009

AMORIM, I. L.; SAMPAIO, E. V. S. B.; ARAÚJO, E. L. Flora e estrutura da vegetação arbustivo-arbórea de uma área de caatinga do Seridó, RN, Brasil Acta Botânica Brasílica, São Paulo, v. 19, n. 3, p. 615-623, 2005.

ANDRADE-LIMA, D. The caatingas dominium. Revista Brasileira de Botânica, São Paulo, v. 4, n. 2, p. 149-153, 1981.

ANDRADE, L. A; OLIVEIRA, F. X.; NEVES, C. M. L.; FÉLIX, L. P. Análise da vegetação sucessional em campos abandonados no agreste paraibano. Revista Brasileira de Ciências Agrárias, Recife, v. 2 , n. 2 , p. 135-142, 2007.

ANDRADE, L. A.; PEREIRA, I. M.; LEITE, U. T.; BARBOSA, M. R. V. Análise da cobertura de duas fitofisionomias de caatinga, com diferentes históricos de uso, no município de São João do Cariri, estado da Paraíba. Cerne, Lavras, v. 11, n. 3, p. 253-262, 2005.

ANGIOSPERM PHYLOGENY GROUP. An update of the angiosperm phylogeny group classification for the orders and families of flowering plants: APG III. Botanical Journal of the Linnean Society, Londres, v. 161, p. 105-121, 2009.

ARAÚJO, E. L.; FERRAZ, E. M. N. Análise da vegetação: amostragem, índices de diversidade e utilidades na etnobotânica. In: ALBUQUERQUE, U. P.; LUCENA R. F. P.; CUNHA, L. V. F. C. (Ed.). Métodos e técnicas na pesquisa etnobotânica. Recife: Comunigraf, 2008. p. 161-198.

ARAÚJO, L. V. C. Composição florística, fitossociológica e influência dos solos na estrutura da vegetação em uma área de caatinga no semi-árido paraibano. 2007. 121 f. Tese (Doutorado em Agronomia) - Universidade Federal da Paraíba, Areia.

BRUMMIT, R. K.; POWELL, C. E. Authors of plant names. Kew: Royal Botanic Gardens, 1992. 732 p.

CALIXTO JÚNIOR, J. T.; DRUMOND, M. A. Estrutura fitossociológica de um fragmento de Caatinga sensu stricto 30 anos após corte raso, Petrolina-PE, Brasil. Revista Caatinga, Mossoró, v. 24, n. 2, p. 67-74, 2011.

CALIXTO JÚNIOR, J. T.; DRUMOND, M. A. ALVES JÚNIOR, F. T. Estrutura e distribuição espacial de Mimosa tenuiflora (Willd.) Poir. em dois fragmentos de Caatinga em Pernambuco. Revista Caatinga, Mossoró, v. 24, n. 2, p. 95-100, 2011.

CIENTEC. Mata nativa: Sistema para análise fitossociológica e elaboração de planos de manejo de florestas nativas. São Paulo, 2002. $126 \mathrm{p}$

COSTA, F. F. Avaliação fitossociológica de um fragmento de caatinga na Bacia Hidrográfica do açude Jatobá, São José do Bonfim, PB. 2008. 49 f. Monografia (Graduação em Engenharia Florestal) - Universidade Federal de Campina Grande, Patos.

DRUMOND, M. A.; KIILL, L. H. P.; NASCIMENTO , C. E. S. Inventário e sociabilidade de espécies arbóreas e arbustivas da Caatinga na Região de Petrolina, PE. Brasil Florestal, v. 21, n. 74, p. 37-43, 2002. 
FABRICANTE, J. R. Estrutura de populações e relações sinecológicas de Cnidoscolus phyllacanthus (Müll. Arg.) Pax \& L. Hoffm. no semi-árido nordestino. 2007. 121 f. Dissertação (Mestrado em Agronomia) - Universidade Federal da Paraíba, Areia.

FABRICANTE, J. R.; ANDRADE, L. A. Análise estrutural de um remanescente de caatinga no Seridó Paraibano. Oecologia Brasiliensis, Rio de Janeiro, v. 3, n. 11, p. 341-349, 2007.

LUNA, R. G. Microbiota edáfica e fitocenose como indicadores de degradação ambiental do semi-árido paraibano. 1998. $98 \mathrm{f}$. Dissertação (Mestrado em Desenvolvimento e Meio Ambiente) Universidade Federal da Paraíba, João Pessoa.

McCune, B. and M. J. Mefford. 1999. PC-ORD. Multivariate analysis of Ecological Data, Version 4.0 for Windows.

MARACAJÁ, P. B.; BATISTA, C. H. F.; SOUSA, A. H. Levantamento florístico e fitossociológico do estrato arbustivoarbóreo de dois ambientes na Vila Santa Catarina, Serra do Mel, RN. Revista de Biologia e Ciências da Terra, Campina Grande, v. 3, n. 2, p. 25-32, 2003.

MARANGON, G. P.; FERREIRA, R. L. C.; SILVA, J. A. L.; LIRA, D. F. S.; SILVA, E. A.; LOUREIRO, G. H. Estrutura e padrão espacial da vegetação em uma área de caatinga. Floresta, Curitiba, v. 43, n. 1, p. 83-92, 2013.

MUELLER-DOMBOIS, D.; ELLEMBERG, H. Aims and methods of vegetation ecology. New York: John Wiley \& Sons, 1974. 547 p.

NASCIMENTO, C. E. S.; RODAL, M. J. N.; CAVALCANTI, A. C. Phytosociology of the remaining xerophytic woodland associated to an environmental gradient at the banks of the São Francisco river - Petrolina, Pernambuco, Brazil. Revista Brasileira de Botânica, São Paulo, v. 26, n. 3, p. 271-287, 2003.

PAYANDEH, B. Comparacion of method for assessing spatial distribution of trees. Forest Science, Bethesda, v. 16, n. 3, p. 312317, 1970.

PEREIRA, I. M.; ANDRADE, L. A.; SAMPAIO, E. V. S. B; BARBOSA, M. R. Use-history effects on structure and flora of caatinga. Biotropica, Lawrence, v. 35, n. 2, p. 154-165, 2003.

PEREIRA JÚNIOR, L. R.; ANDRADE, A. P.; ARAÚJO, K. D. Composição florística e fitossociológica de um fragmento de caatinga em Monteiro, PB. Holos, Natal, v. 28, n. 2, p. 72-84, 2012.

PESSOA, M. F. Estudo da cobertura vegetal em ambientes da caatinga com diferentes formas de manejo no assentamento Moacir Lucena, Apodi, RN. Revista Caatinga, Mossoró, v. 21, n. 3, p. 40-48, 2008.

PRADO, D. E. As caatingas da América do Sul. In: LEAL, I. R.; TABARELLI, M.; SILVA, J. M. C. (Org.). Ecologia e conservação da Caatinga. Recife: Ed. da UFPE, 2003. p. 3-74.
QUEIROZ, J. A.; TROVÃO, D. M. B. M.; OLIVEIRA, A. B.; OLIVEIRA, E. C. S. Análise da estrutura fitossociológica da Serra do Monte, Boqueirão, Paraíba. Revista de Biologia e Ciências da Terra, Campina Grande, v. 6, n. 1, p. 251-259, 2006.

RODAL, M. J. N.; MARTINS, F. R.; SAMPAIO, E. V. S. B. Levantamento quantitativo das plantas lenhosas em trechos de vegetação de caatinga em Pernambuco. Revista Caatinga, Mossoró, v. 21, n. 3, p. 192-205, 2008

SAMPAIO, E. V. S. B. Fitossociologia. In: SAMPAIO, E. V. S. B.; MAYO, S. J.; BARBOSA, M. R. V. (Org.). Pesquisa botânica nordestina: progressos e perspectivas. Recife: Sociedade Botânica do Brasil/Seção Regional de Pernambuco, 1996. p. 203-230.

SAMPAIO, E. V. S. B.; RODAL, M. J. N. Fitofisionomia da Caatinga. In: AVALIAÇÃO e identificação de ações prioritárias para a conservação, utilização sustentável e repartição de benefícios da biodiversidade do bioma Caatinga. Petrolina, 2000. p. 2-14.

SANTANA, J. A. S. Padrão de distribuição e estrutura diamétrica de Croton sonderianus Muell. Arg. (Marmeleiro) na caatinga da Estação Ecológica do Seridó. Revista Verde de Agroecologia e Desenvolvimento Sustentável, Pombal, v. 4, n. 3, p. 85-90, 2009.

SANTANA, J. A. S.; SOUTO, J. S. Diversidade e Estrutura Fitossociológica da Caatinga na Estação Ecológica do Seridó-RN. Revista Brasileira de Ciências da Terra, Campina Grande, v. 6 , n. 2, p. 232-242, 2006.

SANTOS, H. G. dos; JACOMINE, P. K. T.; ANJOS, L. H. C. dos; OLIVEIRA, V. A. de; LUMBRERAS, J. F.; COELHO, M. R.; ALMEIDA, J. A. de; CUNHA, T. J. F.; OLIVEIRA, J. B. de. Sistema brasileiro de classificação de solos. 3. ed. rev. e ampl. Brasília, DF: Embrapa, 2013. 353 p.

SILVA, J. A. Fitossociologia e relações alométricas em caatinga nos estados da Paraíba e Rio Grande do Norte. 2005. 81 f. Tese (Doutorado em Engenharia Florestal) - Universidade Federal de Viçosa, Viçosa, MG.

SISTEMA para análise estatística e genética: manual de uso. Viçosa, MG: UFV/Funarbe, 1997.

SOUTO, P. C. Acumulação e decomposição da serapilheira e distribuição de organismos edáficos em área de caatinga na Paraíba, Brasil. 2006. 150 f. Tese (Doutorado em Agronomia) Universidade Federal da Paraíba, Areia.

SOUZA, J. A. N.; RODAL, M. J. N. Levantamento florístico em trecho de vegetação ripária de caatinga no Rio Pajeú, Floresta/ Pernambuco - Brasil. Revista Caatinga, Mossoró, v. 23, n. 4, p. 54-62, 2010 
